\title{
Proceso de implementación de un programa de habilidades para la vida en adolescentes trabajadores
}

\section{Implementing process of a Life Skills Program for Working Adolescents}

\author{
Jacqueline Gordón Morales $^{1 *}$, Paulina Guerra Guerra ${ }^{1}$ y Sonia Narváez Salazar ${ }^{1}$ \\ ${ }^{1}$ Universidad Tecnológica Indoamérica \\ *jacquelinegordon@uti.edu.ec
}

DOI: https://doi.org/10.26871/killkana_social.v3i2.461

\begin{abstract}
Resumen
El presente documento refleja la primera fase de un proyecto de vinculación con la sociedad en el cual se pretende implementar un programa de habilidades para la vida (OMS), durante el período académico 2018-2019. Se trata de un estudio cuantitativo inferencial con enfoque transversal y con una selección de muestra intencional. En la investigación participaron 82 adolescentes trabajadores de la ciudad de Quito. Se pretende identificar las competencias sociales del grupo y las diferencias entre los adolescentes varones y mujeres, se trata de una metodología eficaz de capacitación en habilidades para la vida como herramienta que facilite una adaptación satisfactoria en el medio en el que se desenvuelven. Esta es una metodología basada en las habilidades para la vida, proporciona un sustento teórico importante en el diseño e implementación de programas de intervención con adolescentes que sirven para la asimilación de habilidades cognitivas y sociales dotándole a los adolescentes de recursos que le sirvan para afrontar los retos que les depara la vida. Para la medición de las competencias sociales se usa la prueba de "Actitudes y Estrategias Cognitivas Sociales" (AECS) mediante el análisis comparativo de medias de las 19 sub-escalas que contiene el reactivo. Se presentan los resultados de la primera evaluación en la que se encontraron diferencias estadísticamente significativas entre varones y mujeres en 5 escalas: agresividad, dominancia, divergencia/convergencia, habilidad/inhabilidad para anticipar y comprender las consecuencias que posiblemente seguirán de los comportamientos sociales y habilidad/inhabilidad para elegir los medios adecuados a los fines que se persiguen en el comportamiento social y en la percepción del ejercicio democrático/autoritario de la disciplina de los padres.
\end{abstract}

Palabras clave: Actitud, comportamiento, contexto, habilidades para la vida, habilidades sociales.

\begin{abstract}
This study presents the first stage of a relationship project aimed to implement a life skills program (WHO) during the 2018-2019 academic year. This is a quantitative inferential study with a cross-sectional approach and a planned sample selection. Eighty-two adolescent workers from the city of Quito participated in this research. The goal is to identify the group's social competencies and the differences between male and female adolescents. This is a life skills' training methodology which is very effective as a tool that facilitates satisfactory adaptability in their functioning environment. This life skills-based methodology provides important theoretical support in the design and implementation of intervention programs with adolescents and facilitates the assimilation of cognitive and social skills giving youngsters helpful resources so they could face the challenges that life has to offer. For measuring social competences, the "Social Cognitive Attitudes and Strategies" (AECS) test is used through the application of a comparative analysis of means, of the 19 subscales contained in the experiment. The results of the first evaluation, in which statistically significant differences were found between men and women, are shown in 5 scales: 1. aggressiveness, 2. dominance, 3. divergencelconvergence, 4. ability/inability to anticipate and understand the likely social behaviors consequences and 5. Ability/inability for choosing the appropriate means to achieve the pursued goals in social behavior and in democratic/authoritarian perception exercise of parental discipline.
\end{abstract}

Key words: Attitude, behavior, context, life skills, social skills.

\section{Introducción}

La competencia social tiende a ser vista desde diferentes aristas; una versión general la describe como un conjun- to de habilidades sociales que facilitan la adaptación de la persona a un contexto social determinado. López, Iriarte y González (2004), desde una visión más amplia y compleja, 
indican que la competencia social se basa en las relaciones entre personas, estas relaciones integran procesos de pensamientos, sentimientos y conductas que finalmente se traducen en el uso adecuado de las habilidades sociales con base en el entendimiento de las situaciones interpersonales. Un entorno favorable de interacción social también facilita la relación social. Desde otro punto de vista, Camacho (2005) habla de la imposibilidad de definir a la competencia social porque esta es, en parte, dependiente de un contexto que varía.

Estas definiciones llegan a un significativo punto de convergencia en cuanto a la relevancia del contexto como el caldo de cultivo donde se evidencian los comportamientos sociales como forma de adaptación.

Dentro de los factores contextuales influyentes en la competencia o incompetencia social, según Martínez (2007), el hogar y el centro educativo son importantes condicionantes en la forma en la que socializan los adolescentes y son complementarias a las características de personalidad. Con esto se reafirma la idea de que la competencia social no es una fórmula estática en la persona, las instituciones educativas pueden ser fuente de perfeccionamiento de habilidades cognitivas y sociales.

En este contexto, la Organización Mundial de la Salud (OMS) propone el término de habilidades para la vida que toma auge desde los años 90. Según Bravo (2005), el objetivo de educar en habilidades para la vida es que las nuevas generaciones se apropien de herramientas psicológicas y sociales que de alguna forma predigan un estilo de vida saludable.

El tiempo trascurre y las características de las personas adaptándose a las nuevas demandas del contexto requieren también cambios de enfoque direccionados a la práctica más allá del mero entendimiento. La OMS (1993) aclara que el tema relacionado con las habilidades para la vida se venía desarrollando desde los años sesenta en donde los facilitadores proporcionaban información de lo perjudiciales que podrían ser ciertos comportamientos y a través de eso se esperaba que las personas reduzcan comportamientos en función del nuevo conocimiento. Este paradigma obedece a un tipo de educación tradicional en donde se depositan conocimientos y se esperan comportamientos en función de la recompensa o el castigo.

El enfoque contemporáneo de la enseñanza de estas habilidades propone el desarrollo y fortalecimiento de diez competencias básicas esenciales: "manejo de emociones y sentimientos, empatía, relaciones interpersonales, comunicación efectiva, pensamiento creativo, pensamiento crítico, toma de decisiones, solución de problemas y conflictos, conocimiento de sí mismo y manejo de estrés" (World Health Organization., 1994, p.24). Esta propuesta considera los desafíos que tienen los adolescentes en la sociedad y las complejidades de la globalización, las tecnologías y la interacción social.

En una experiencia de implementación de un programa de habilidades para la vida en Perú, Chirinos (2009), concluyó que la autoestima y la habilidad de toma de decisiones no manifestaron diferencias significativas entre una primera y segunda evaluación. Los autores concluyen que el trabajo en la autoestima requiere de mayor temporalidad pues requiere de otras habilidades para consolidarse (auto concepto, autopercepción, autoevaluación). Esta investigación generó una necesidad de reorientar la implementación del programa que, en este caso, está a cargo de Ministerio de Salud de Perú.

El reto entonces vendría a ser la forma en la que se puede lograr esta apropiación de habilidades de vida, es decir, cuál sería la metodología contextualizada que logre potenciar el estilo de vida de los adolescentes y la medición de la efectividad de la misma para un constante proceso de mejoramiento.

\section{Evaluación de competencia social}

En el modelo teórico de competencia social de los adolescentes que plantean Moraleda, González y García (1998), se establecen variables actitudinales y cognitivas que en un continuo de tiempo determinan competencia o incompetencia social. Las variables actitudinales presentan una estructura trifactorial y bipolar. En la estructura trifactorial "cabe distinguir un factor prosocial o facilitador de las relaciones, otro antisocial o destructor de las relaciones y otro asocial o inhibidor ante las relaciones" (Moraleda y cols., 1998, p.7). En el factor prosocial se encuentra la solidaridad y al liderazgo; dentro de la solidaridad están los aspectos de conformidad con lo que es socialmente correcto, sensibilidad social, ayuda y colaboración, y seguridad y firmeza en la interacción. El factor antisocial abarca la dominancia y agresividad-terquedad. El factor asocial incluye los factores de retraimiento y ansiedad-timidez.

La estructura bipolar de las variables actitudinales sitúa a las variables antes mencionadas en dos polos, uno positivo, en el que se ubican las que corresponden al factor prosocial y un polo negativo en el que se integran los factores antisociales y asociales. Estos dos polos marcan la competencia o incompetencia social, mientras el factor prosocial promueve la adaptación mediante la interacción adolescente, el factor antisocial y asocial limitan los procesos de interacción.

El modelo teórico de competencia social del test AECS, además de variables actitudinales, establece también variables cognitivas.

Las variables cognitivas más vinculadas al éxito o fracaso de la relación social de los adolescentes se agrupan en tres factores: uno relacionado con la percepción social de los adolescentes, otro con sus estilos cognitivos y otro con las estrategias cognitivas empleadas en la solución de problemas sociales. (Moraleda y cols., 1998, p.8)

Estas variables cognitivas también presentan una estructura bipolar. En el polo positivo se encuentran los factores cognitivos que promueven la interacción social y, por ende, se acercan a la competencia social; en oposición, los procesos cognitivos que dificultan la relación social 
adolescente en el polo negativo. En el polo positivo se sitúan procesos cognitivos relacionados con la percepción (percepción y expectativas positivas sobre las relaciones y percepción del clima social del hogar como democrático y acogedor) estilos cognitivos (reflexividad, dependencia de campo y convergencia) estrategias cognitivas empleadas en la solución de problemas sociales (habilidad en la observación y retención de información, búsqueda de alternativas, anticipación ante consecuencias y elección de medios adecuados de solución) (Moraleda y cols., 1998).

En la Tabla 1 se puede evidenciar la distribución bipolar de las variables. En el polo negativo están las estrategias cognitivas opuestas a las del polo positivo, por ejemplo, en las variables relacionadas a las estrategias en la resolución de problemas sociales encontramos a la inhabilidad en la búsqueda de alternativas en el polo negativo versus la habilidad en la búsqueda de alternativas en el polo positivo.

Tabla 1 Estructura bipolar de la competencia social de los adolescentes

\begin{tabular}{|c|c|c|}
\hline & $\begin{array}{c}\text { Competencia social Polo } \\
\text { positivo }\end{array}$ & $\begin{array}{l}\text { Incompetencia social } \\
\text { Polo negativo }\end{array}$ \\
\hline \multirow[t]{4}{*}{$\begin{array}{c}\text { Variables } \\
\text { actitudinales }\end{array}$} & $\begin{array}{l}\text { Conformidad con lo que es } \\
\text { socialmente correcto }\end{array}$ & Dominancia \\
\hline & Sensibilidad social & Agresividad-terquedad \\
\hline & Ayuda y colaboración & Apatía-retraimiento \\
\hline & $\begin{array}{l}\text { Seguridad y firmeza en la in- } \\
\text { teracción }\end{array}$ & Ansiedad-timidez \\
\hline \multirow{10}{*}{$\begin{array}{l}\text { Variables } \\
\text { cognitivas }\end{array}$} & Reflexividad & Impulsividad \\
\hline & Dependencia de campo & Independencia de campo \\
\hline & Divergencia & Convergencia \\
\hline & $\begin{array}{l}\text { Percepción y expectativas } \\
\text { positivas }\end{array}$ & $\begin{array}{l}\text { Negativas sobre la relación } \\
\text { social }\end{array}$ \\
\hline & $\begin{array}{l}\text { Percepción ejercicio demo- } \\
\text { crático }\end{array}$ & $\begin{array}{l}\text { Autoritario de la disciplina } \\
\text { de los padres }\end{array}$ \\
\hline & $\begin{array}{l}\text { Percepción actitud acogedo- } \\
\text { ra y benevolente }\end{array}$ & $\begin{array}{l}\text { Rechazante y hostil de los } \\
\text { padres }\end{array}$ \\
\hline & $\begin{array}{l}\text { Habilidad en la observación } \\
\text { y retención de información }\end{array}$ & $\begin{array}{l}\text { Inhabilidad en la observa- } \\
\text { ción y retención de informa- } \\
\text { ción }\end{array}$ \\
\hline & $\begin{array}{l}\text { Habilidad en la búsqueda de } \\
\text { alternativas }\end{array}$ & $\begin{array}{l}\text { Inhabilidad en la búsqueda } \\
\text { de alternativas }\end{array}$ \\
\hline & $\begin{array}{l}\text { Habilidad en la anticipación } \\
\text { de consecuencias }\end{array}$ & $\begin{array}{l}\text { Inhabilidad en la anticipa- } \\
\text { ción de consecuencias }\end{array}$ \\
\hline & $\begin{array}{l}\text { Habilidad en la elección de } \\
\text { medios adecuados }\end{array}$ & $\begin{array}{l}\text { Inhabilidad en la elección de } \\
\text { medios adecuados }\end{array}$ \\
\hline
\end{tabular}

Nota: Adaptado de "AECS, Actitudes y Estrategias Cognitivas Sociales", (Moraleda y cols., 1998)

La competencia o incompetencia social no se limita a tener o carecer de un número de habilidades de adaptación que se necesitan en una situación social particular, "una persona puede ser incompetente socialmente no porque le falten las habilidades conductuales, sino porque no las ponga en juego debido a que factores emocionales, cognitivos y/o motores interfieren en su ejecución" (López de y cols., 2004, p.148). De aquí surge la opción de realizar procesos de intervención en el contexto como una estrategia para mejorar la competencia social en los adolescentes y, por lo tanto, sus procesos adaptativos. Lacunza (2011) resalta la importancia de las características de los padres, la experiencia en crianza y el acceso a televisión en internet como contextos o situaciones sociales decisivas para el aprendizaje de habilidades sociales funcionales o disfuncionales. Según Muñoz, Carreras y Braza (2004) es posible prevenir riesgos relacionados a conductas antisociales y de inadaptación escolar, violencia, racismo e intolerancia si se relaciona a la competencia social con los procesos de adaptación en el contexto académico.

García, Cabanillas, Moran y Olaz (2014) señalan que los adolescentes son socialmente presionados para cumplir estereotipos determinados por la cultura y son transferidos por la familia, entornos escolares y medios de comunicación, para poder adaptarse como hombres y mujeres en su medio social, se indica, por ejemplo, que se espera que los hombres sean más asertivos y las mujeres más gentiles. En cuanto a habilidades sociales, se asigna al hombre a aquellas relacionadas con la expresión de sentimientos negativos, y en lo que respecta al "rol femenino por otra parte estaría relacionado con características como la crianza, cuidado, sensibilidad y apertura emocional, mayor fortaleza emocional, y habilidades sociales vinculadas a la expresión de sentimientos positivos, la empatía y la aceptación” (García M. Cabanillas G. Moran V. Olaz, 2014, p.116).

Diversos estudios sustentan la presencia de diferencias en las habilidades sociales de varones y mujeres, sin embargo, no se evidencia una estructura diferencial de aspectos específicos de competencia social de la adolescencia en ambos sexos, y que aporte al abordaje contextual de dificultades en la socialización con base en el modelo de competencia social.

\section{Método}

En esta primera parte de la investigación se utilizó una metodología cuantitativa de carácter descriptivo con enfoque transversal que tiene como objetivo identificar diferencias estadísticas significativas entre hombres y mujeres, respecto a las variables relacionadas con habilidades sociales presentes en las 19 escalas del test AECS (Actitudes y estrategias cognitivas sociales). Se tomaron como criterio de comparación las actitudes y estrategias cognitivas sociales de un grupo de adolescentes del Centro del Muchacho Trabajador.

La hipótesis H1 planteada en el estudio establece diferencias de puntuación entre el género masculino y femenino en referencia a las actitudes y estrategias cognitivo sociales, mientras que la $\mathrm{H} 0$ plantea igualdad de puntuaciones en referencia a las actitudes y estrategias cognitivo sociales entre el género masculino y femenino.

Para descartar HO (hipótesis nula) de igualdad de criterios entre ambos sexos respecto a las actitudes y estrategias cognitivo sociales se utilizó la prueba no paramétrica U de Mann Whitney.

La muestra estuvo constituida por 81 estudiantes de educación secundaria provenientes del Centro del Muchacho Trabajador. De la muestra, 40 eran mujeres $(48,80 \%)$ y 41 varones $(52,20 \%)$, con una edad comprendida entre $11 \mathrm{y}$ 
13 años. En la Figura 1 se muestran varios participantes del proyecto en una de las actividades guiadas por estudiantes. El muestreo fue intencional con participación voluntaria.

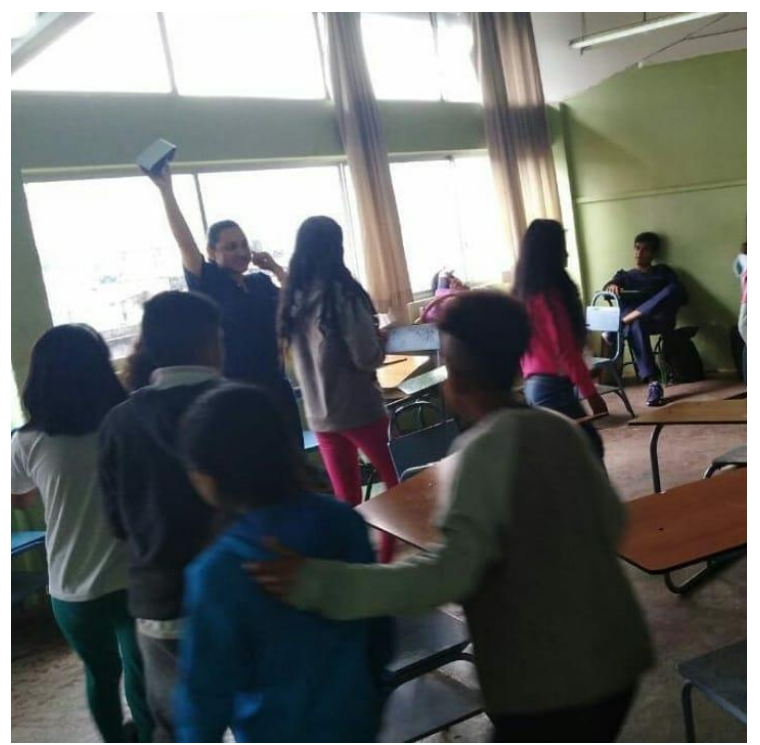

Figura 1. Grupo de adolescentes participando en una actividad guiada

El instrumento utilizado en la investigación fue el AECS (Moraleda et al., 1998). Es un cuestionario que se basa en el modelo teórico de competencia social que evalúa nueve actitudes sociales y diez estrategias de pensamiento social que se distribuyen de la siguiente manera:

- Escalas de actitudes sociales: conformidad con lo socialmente correcto (Con), sensibilidad social (Sen), ayuda y Colaboración (Ac), seguridad y firmeza en la interacción (Sf), liderazgo prosocial (Lid), agresividadterquedad (Agr), dominancia (Dom), apatía retraimiento (Ap), ansiedad-timidez (Ans).

- Escalas de pensamiento social: reflexividad/impulsividad (Imp), dependencia/independencia de campo (Ind), divergencia/convergencia (Cv), percepción y expectativas positivas/negativas sobre la relación social (Per), percepción ejercicio democrático/autoritario de la disciplina de los padres (Dem), percepción actitud acogedora y benevolente/rechazante y hostil de los padres (Hos), habilidad/inhabilidad en la observación y retención de la información relevante sobre las situaciones sociales (Obs), habilidad/inhabilidad en la búsqueda de soluciones alternativas para resolver los problemas sociales (Alt), habilidad/inhabilidad para anticipar y comprender las consecuencias que posiblemente se seguirán de los comportamientos sociales (Cons), habilidad/inhabilidad para elegir los medios adecuados a los fines que se persiguen en el comportamiento social (Med)

\section{Resultados}

En función de analizar los resultados de la primera evaluación de actitudes y estrategias cognitivas sociales, se evidencia en la Tabla 2 la diferenciación de rangos promedio entre hombres y mujeres en las escalas Agr, Dom, $\mathrm{Cv}$, Cons, Med y Dem. Aquí se evidencia un mayor rango promedio en varones en las seis escalas mencionadas.

Tabla 2 Comparación por sexos de las puntuaciones medias en las actitudes y estrategias cognitivas sociales

\begin{tabular}{|c|c|c|c|c|}
\hline $\begin{array}{l}\text { Actitudes } \\
\text { y estrategias } \\
\text { cognitivo sociales }\end{array}$ & Sexo & $\mathrm{N}$ & $\begin{array}{c}\text { Rango } \\
\text { promedio }\end{array}$ & $\begin{array}{c}\text { Suma de } \\
\text { rangos }\end{array}$ \\
\hline \multirow{3}{*}{$\begin{array}{l}\text { Agresividad/ } \\
\text { Terquedad (Agr) }\end{array}$} & femenino & 39 & 34.51 & 1346.00 \\
\hline & masculino & 41 & 46.20 & 1894.00 \\
\hline & Total & 80 & & \\
\hline \multirow{3}{*}{$\begin{array}{l}\text { Dominancia } \\
\text { (Dom) }\end{array}$} & femenino & 39 & 34.95 & 1363.00 \\
\hline & masculino & 41 & 45.78 & 1877.00 \\
\hline & Total & 80 & & \\
\hline \multirow{3}{*}{$\begin{array}{l}\text { Divergencia/Convergencia } \\
\text { (Cv) }\end{array}$} & femenino & 40 & 34.43 & 1377.00 \\
\hline & masculino & 41 & 47.41 & 1944.00 \\
\hline & Total & 81 & & \\
\hline \multirow{3}{*}{$\begin{array}{l}\text { Habilidad/inhabilidad para } \\
\text { anticipar y comprender las } \\
\text { consecuencias que } \\
\text { posiblemente se seguirán de } \\
\text { los comportamientos sociales } \\
\text { (Cons) }\end{array}$} & femenino & 40 & 33.16 & 1326.50 \\
\hline & masculino & 41 & 48.65 & 1994.50 \\
\hline & Total & 81 & & \\
\hline \multirow{3}{*}{$\begin{array}{l}\text { Habilidad/inhabilidad para } \\
\text { elegir los medios adecuados a } \\
\text { los fines que se persiguen en el } \\
\text { comportamiento social (Med) }\end{array}$} & femenino & 40 & 35.26 & 1410.50 \\
\hline & masculino & 41 & 46.60 & 1910.50 \\
\hline & Total & 81 & & \\
\hline \multirow{3}{*}{$\begin{array}{l}\text { Percepción ejercicio } \\
\text { democrático/autoritario de la } \\
\text { disciplina de los padres (Dem) }\end{array}$} & femenino & 40 & 35.35 & 1414.00 \\
\hline & masculino & 41 & 46.51 & 1907.00 \\
\hline & Total & 81 & & \\
\hline
\end{tabular}

En la Tabla 3 se evidencia que se puede rechazar la hipótesis nula en las escalas Agr, Dom, Cv, Cons, Med y Dem que indican diferencias estadísticamente significativas.

Tabla 3 Nivel de significancia según la prueba $U$ de Mann-Whitney

\begin{tabular}{|c|c|c|c|c|c|c|}
\hline & Agr & Dom & Med & Dem & Cons & $\mathrm{Cr}_{\mathrm{V}}$ \\
\hline U de Mann-Whitney & 566.000 & 583.000 & 590.500 & 594.000 & 506.500 & 557.000 \\
\hline W de Wilcoxon & 1346.000 & 1363.000 & 1410.500 & 1414.000 & 1326.500 & 1377.000 \\
\hline$z$ & -2.250 & -2.087 & -2.170 & -2.138 & -2.964 & -2.490 \\
\hline Sig. asintótica (bilateral) & .024 & .037 & .030 & .033 & .003 & .013 \\
\hline
\end{tabular}

Nota. ${ }^{*} p<0,05,{ }^{*} p<0,01$

La diferencia en las puntuaciones de Agresividad/Terquedad (Agr) más altas en los varones (incompetencia social) que en las mujeres, fue significativa, $\mathrm{U}=$ $2.250, p=0.024$ ( $p<.05)$, con 46.20 para varones y 34.51 para mujeres. En cuanto a las puntuaciones de Dominancia (Dom), estas fueron más altas en los varones (incompetencia social) que en las mujeres, esta diferencia fue significativa, $\mathrm{U}=-2.087, \mathrm{p}=0.037(\mathrm{p}<.05)$, con 45.78 para varones y 34.95 para mujeres. En lo que tiene que ver con Divergencia/ Convergencia $(\mathrm{Cv})$, las puntuaciones son más altas en los varones (competencia social) que en las mujeres, esta diferencia fue significativa, $\mathrm{U}=-2.490, \mathrm{p}=0.013$ ( $\mathrm{p}<.05$ ), con 47.41 para varones y 34.43 para mujeres. En Habilidad/inhabilidad para anticipar y comprender las 
consecuencias que posiblemente se seguirán de los comportamientos sociales (Cons), las puntuaciones son más altas en los varones (competencia social) que en las mujeres, esta diferencia fue significativa, $\mathrm{U}=-2.964, \mathrm{p}=0.003(\mathrm{p}<.01)$, con 48.65 para varones y 33.16 para mujeres. En referencia a Habilidad/inhabilidad para elegir los medios adecuados a los fines que se persiguen en el comportamiento social (Med), las puntuaciones son más altas en los varones (competencia social) que en las mujeres, esta diferencia fue significativa, $\mathrm{U}=-2.170, \mathrm{p}=0.030(\mathrm{p}<.05)$, con 46.69 para varones y 35.26 para mujeres. Finalmente, Percepción ejercicio democrático/autoritario de la disciplina de los padres (Dem), las puntuaciones son más altas en los varones (competencia social) que en las mujeres, esta diferencia fue significativa, $\mathrm{U}=-2.138, \mathrm{p}=0.033(\mathrm{p}<.05)$, con 46.51 para varones y 35.35 para mujeres.

\section{Conclusiones}

La adolescencia es un tiempo crucial para formar una imagen coherente y ajustada de la forma de ser de la persona y que coincide con la adquisición de habilidades cognitivas que, consecuentemente, facilitarán su desempeño social, según Pastor, García \& Balaguer (2003). Partiendo de esta premisa, y en cumplimiento del objetivo de investigación, la discusión se centra en las diferencias entre varones y mujeres en estas formas de ajuste propio para la acomodación social mediante el uso del test AECS.

Se encontraron cuatro escalas con diferencias significativas, dos pertenecen a las escalas de actitud social: agresividad/terquedad (Agr), dominancia (Dom), y cuatro pertenecen a las escalas de pensamiento social: divergencia/convergencia $(\mathrm{Cv})$, habilidad/inhabilidad para anticipar y comprender las consecuencias que posiblemente se seguirán de los comportamientos sociales (Cons), habilidad/inhabilidad para elegir los medios adecuados a los fines que se persiguen en el comportamiento social (Med) y percepción ejercicio democrático/autoritario de la disciplina de los padres (Dem).

La puntuación elevada en las escalas de agresividad y dominancia en adolecentes varones (polo negativo) ubica esta tendencia dentro del factor antisocial. Se describe como la predisposición para intimidación, inflexibilidad, buscar ser autoridad, dominar a las otras personas para sus propios fines y competir con otros con habilidades de manipulación para obtener beneficios. Esta particularidad, de alguna manera se encuentra justificada a partir de los estereotipos que ubican al hombre como dominante sobre la mujer, con fortaleza física y emocional que le respaldan, "la negación de las emociones en la socialización de los chicos dificulta que éstas se mediaticen a través de la palabra por lo que se tiende a recurrir a la fuerza del cuerpo o a la imposición para resolver los conflictos" (Benlloch, Sanchez, y Valencia, 2008, p.110). Estas manifestaciones de poder en los adolescentes varones se encuentran de alguna manera apoyadas en las expectativas sociales del ser hombre. Estas relaciones asimétricas se basan en los modelos de relación y estereotipos tradicionales que, a pesar de que se encuentra incorporada la educación mixta (coeducación), no se evidencia la incorporación de estrategias de socialización igualitaria como eje transversal de educación.

En cuanto a las escalas de pensamiento social, las puntuaciones elevadas en divergencia/convergencia, habilidad/inhabilidad para anticipar y comprender las consecuencias que posiblemente se seguirán de los comportamientos sociales, habilidad/inhabilidad para elegir los medios adecuados a los fines que se persiguen en el comportamiento social y percepción ejercicio democrático/autoritario de la disciplina de los padres. Las características de las elevaciones en estas escalas obedecen a una forma de pensamiento rígido, autoritario, con dificultad para buscar alternativas a los problemas, no medir las consecuencias de los propios actos, no anticipar obstáculos y en el ambiente familiar se puede expresar libremente, es escuchado y se acepta su modo de pensar (Moraleda y cols., 1998).

La autodescripción de las y los adolescentes es un reflejo del contexto social en el que se desarrollan, es así que Benlloch et al., (Benlloch y cols., 2008, year) resalta que la identidad femenina sigue siendo representada por la dependencia emocional, la comprensión y cuidado de los demás, mientras que la masculina se define por la racionalidad, control emocional y dominancia. Las actitudes hostiles de los hombres hacia las mujeres evidencian el control que estos intentan tener sobre el sexo opuesto (VelandiaMorales y Rodríguez-Bailón, 2011); esta situación ubica a las mujeres en un grupo subordinado. Las actitudes dominantes de los hombres se proyectan sobre las mujeres reproduciendo los roles tradicionales de lo masculino y lo femenino que aún se mantienen en el contexto socio cultural actual. Así, mientras que en los adolescentes se incentiva la defensa de sus propios derechos y la expresión de desagrado y comportamiento competitivo-agresivo, a las adolescentes se les inculca el anteponer las necesidades de otros ante las propias, ceder ante la iniciativa masculina y la supresión de necesidades que se incorporan en la forma en que se comportan en el medio social y familiar (Inglés, Martínez-Monteagudo, Delgado, Torregrosa, Redondo, Benavides, García-López, 2008).

Estas formas de relación social que se mantienen en el tiempo, según Rey (2008), se relacionan con los patrones de comportamiento de los padres y otras personas significativas en sus vidas, esto transforma a los comportamientos dominantes y de sumisión admisibles en su forma de socializar. Según Vallejo (2006), las características autoatribuidas de adolescentes, hombres y mujeres obedecen todavía a una representación sexista del mundo. Los niños y niñas se van identificando con roles, cualidades $\mathrm{y}$ hasta problemas previstos para uno $\mathrm{u}$ otro género, es así que se puede percibir en la realidad diaria y aceptada hombres insensibles, competitivos, duros y mujeres tiernas, empáticas, dependientes y pasivas como producto de la sociedad y cultura que, en este caso, no se puede asumir 
como fortaleza sino como debilidad en el momento de identificarse y posicionarse socialmente desde la igualdad.

Este estudio contextualizado de la población permite un proceso de intervención desde el conocimiento de la realidad del grupo y sus necesidades, Cardozo, Dubini, Fantino y Ardiles (2011), resaltan las evidencias de la efectividad del enfoque de habilidades para la vida como mediador en la apropiación y construcción de normas sociales desde la premisa de enseñarles a pensar, no qué pensar, de empoderamiento en la toma de decisiones y control de sus propias emociones.

La metodología para los talleres de habilidades para la vida prioriza al diálogo, la horizontalidad, participación y crecimiento mutuo de las personas, los procesos comunicativos dejan de ser depositarios y se valoran las experiencias personales para la apropiación de conocimientos construidos desde las vivencias personales que involucran el desarrollo tecnológico, la globalización, los cambios en la interacción social y la propia estructura familiar. En la Figura 2 se evidencia uno de los momentos de interacción del proyecto de vinculación con la sociedad.

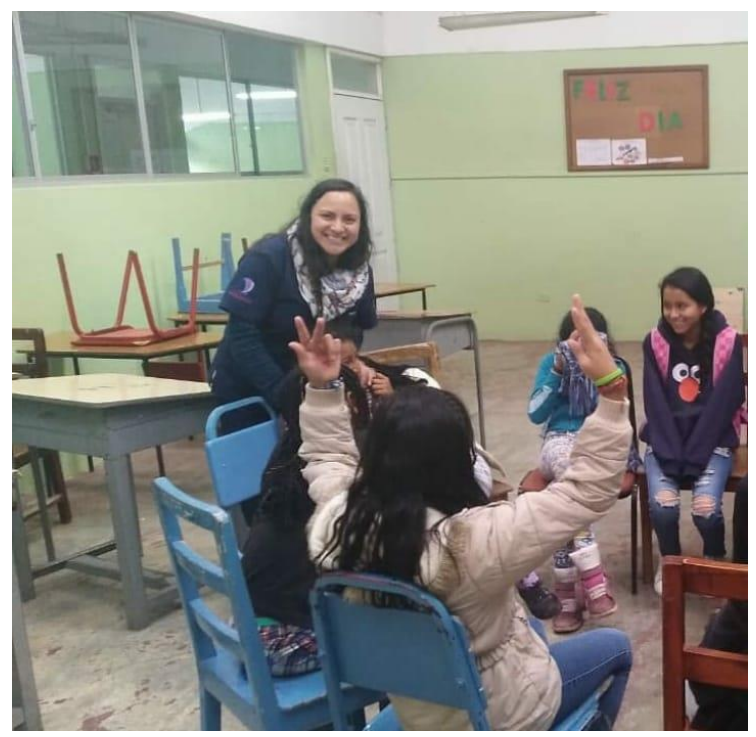

Figura 2. Interacción en proyecto de Vinculación

\section{Recomendaciones}

En consideración, con las limitaciones de esta fase es recomendable ampliar el estudio a otras poblaciones para suprimir variables intervinientes propias de la muestra seleccionada. Así mismo, sería importante que se realice un estudio evolutivo longitudinal para establecer cómo se da el proceso de adquisición de las conductas adaptativas atribuidas a los estereotipos de hombres y mujeres, y luego contrastar los resultados del test AECS con otros instrumentos de evaluación de competencia social o un instrumento que evalúe las habilidades para la vida.

\section{Agradecimiento}

Al Centro del Muchacho Trabajador y a los estudiantes de la Universidad Indoamérica, Sede Quito, Carrera de
Psicología, integrantes del Proyecto de Vinculación: Sonia Narváez, Nicole Sánchez, Fidel Bonilla, Alisson Herrera, Sara Albuja, Jesús Moreno, Daniela Palacios, Giselt Quintero, Vanessa Viera, Vanessa Ordoñez, Ricardo Palacios, Alexandra Pillajo, José David Salcedo, Angie Vaca, Vanessa Villarreal y Coralía Macías.

\section{Referencias Bibliográficas}

Benlloch, I. M., Sanchez, L. G., y Valencia, U. D. (2008). Identidad de género y afectividad en la adolescencia: asimetrías relacionales y violencia simbólica. Аnиаrio de Psicología, 39, 109-118.

Bravo, A. (2005). La iniciativa de habilidades para la vida en el ámbito escolar. Revista del Instituto de investigaciones Educativas, 9(16), 25-34.

Camacho, C., y Camacho, M. (2005). Habilidades sociales en adolescencia: un programa de intervención. Revista Profesional Española de Terapia CognitivoConductual, 3, 1-27.

Chirinos-C, L. (2009). Eficacia del programa de habilidades para la vida en adolescentes escolares de huancavelica. Perú, 11(2), 169-181.

García M. Cabanillas G. Moran V. Olaz, F. (2014). Diferencias de género en habilidadessociales en estudiantes universitarios de argentina. Anuario Electrónico de Estudios En Comunicación Social, 7, 114-135.

López de, D. R., Iriarte Redín, C., y González Torres, C. (2004). Aproximación y revisión del concepto "competencia social”. Revista Española de Pedagogía, 62(227), 143- 156.

Martínez, V. (2007). La buena educación. En E. Barcelona (Ed.), Reflexiones y propuestas de psicopedagogía humanista. Anthropos Editorial.

Moraleda, M., González, A., y García, J. (1998). Aecs, actitudes y estrategias cognitivas sociales. Madrid, España: TEA Ediciones S.A.

Rey, C. (2008). Habilidades pro sociales, rasgos de personalidad de género y aceptación de la violencia hacia la mujer, en adolescentes que han presenciado violencia entre sus padres. Acta Colombiana de Psicología, 11(1), 107-118. Descargado de http://www.scielo.org.co/pdf/acp/ v11n1/v11n1a11.pdf

Ruiz, P., Y., G. M., L., M., y Balaguer Solá, I. (2003). El autoconcepto y la autoestima en la adolescencia media: análisis diferencial por curso y género. Revista de Psicología Social, 18(2), 141-162. Descargado de https://doi.org/10.1174/ 021347403321645258

Velandia-Morales, A., y Rodríguez-Bailón, R. (2011). Estereotipos femeninos y preferencia de consumo. Universitas Psychologica, 10(1), 47-60. 
Recibido: 5 de mayo de 2019

Aceptado: 25 de junio de 2019

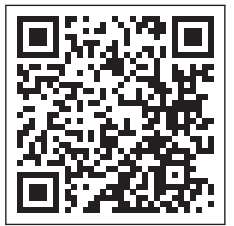


